\title{
Serious Games Go Informal: A Museum-Centric Perspective on Intelligent Game-Based Learning
}

\author{
Jonathan P. Rowe ${ }^{1}$, Eleni V. Lobene, Bradford W. Mott and James C. Lester \\ Department of Computer Science, North Carolina State University, Raleigh, NC 27695 \\ \{jprowe, eavagias, bwmott, lester\}@ncsu.edu
}

\begin{abstract}
Intelligent game-based learning environments show considerable promise for creating effective and engaging learning experiences that are tailored to individuals. To date, much of the research on intelligent game-based learning environments has focused on formal education settings and training. However, intelligent game-based learning environments also offer significant potential for informal education settings, such as museums and science centers. In this paper, we describe FUTURE WORLDS, a prototype game-based learning environment for collaborative explorations of sustainability in science museums. We report findings from a study investigating the influence of individual differences on learning and engagement in FUTURE WORLDS. Results indicate that learners showed significant gains in sustainability knowledge as well as high levels of engagement. Boys were observed to actively engage with FUTURE WORLDS for significantly longer than girls, and young children engaged with the exhibit longer than older children. These findings support the promise of intelligent game-based learning environments that dynamically recognize and adapt to learners' individual differences during museum learning.
\end{abstract}

Keywords: Intelligent game-based learning environments, informal science education, individual differences, science museums, educational games.

\section{Introduction}

There is growing evidence suggesting that game-based learning environments are effective educational tools [1]. Intelligent game-based learning environments, which integrate rich, immersive experiences of digital games with adaptive pedagogical functionalities of intelligent tutoring systems, offer considerable promise [2-4]. To date, much of the research on intelligent game-based learning environments has focused on formal education settings [1-3] and training [4]. However, informal education settings, such as museums and science centers, stand poised to benefit as much, or perhaps even more so, from advances in intelligent tutoring and game-based learning technologies $[5,6]$. While the goals of formal education and informal education settings overlap, informal science education places particular emphasis on

\footnotetext{
${ }^{1}$ Corresponding Author: Jonathan P. Rowe, Department of Computer Science, North Carolina State University
} 
affective and attitudinal outcomes, which have significant implications for the design of intelligent game-based learning environments [6].

In this paper, we investigate how learners' individual differences impact learning and engagement during game-based learning, as well as how individual differences should influence the design of museum-centric intelligent game-based learning environments. To investigate these questions we have developed FUTURE WORLDS, a prototype game-based learning exhibit for sustainability education in science museums. We report findings from a museum-based study that suggest FUTURE WORLDS is effective at fostering significant gains in sustainability understanding and high levels of engagement. In addition, results indicate that the exhibit elicits more extended durations of engagement among boys and young children than girls and older children. Based on these findings, we argue that intelligent game-based learning environments in museums should incorporate automated detector models for recognizing learners' individual differences, as well as pedagogical planners that tailor problem scenarios based on these characteristics.

\section{FUtURE WORLDS}

FUTURE WORLDS is a prototype game-based learning environment about environmental sustainability designed for children ages 9-12 [6]. The exhibit integrates game-based learning environments, intelligent tutoring systems, and interactive tabletop displays to enable collaborative explorations of environmental sustainability. Learners solve sustainability-centered problem scenarios by investigating alternate environmental decisions in a 3D simulated environment (Fig. 1). The effects of learners' decisions are realized in real-time through vibrant $3 \mathrm{D}$ graphics, and they are accompanied by narrated explanations from a robot-like animated pedagogical agent.

The prototype exhibit consists of two integrated displays: a horizontally oriented Samsung SUR40 interactive tabletop, and a vertically oriented 50" high-definition television. Visitors congregate around the interactive tabletop to explore the simulation through multi-touch interactions. The vertical display provides additional space for explanations of sustainability, which are accessible to learners standing farther away from the exhibit. FUTURE WORLDS' 3D environments and sustainability simulation are built with the Unity game engine.

Building on sustainability curricula, such as Facing the Future's Global Sustainability Resources, the FUTURE WORLDS curriculum focuses on three integrated themes of sustainability: water, food, and energy. Visitors' objective during learning interactions with the FUTURE WORLDS exhibit is to use the interactive tabletop display to

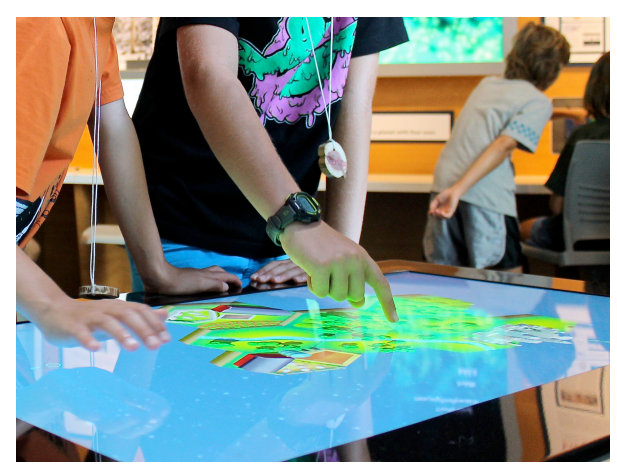

Fig. 1. Future WORLDS exhibit. 
collaboratively (or individually) reconfigure an unsustainable virtual environment into a sustainable environment. The first sustainability-centered problem scenario focuses on a virtual watershed that is plagued by several examples of unsustainable farming practices. Learners explore potential solutions to the environment's sustainability challenges, such as incorporating renewable energy sources into the region's electricity portfolio, implementing organic farming practices, and instituting novel waste-to-energy technologies. As learners manipulate the environment, their choices are visualized in real-time through the environment's 3D models, textures, and animations, and the changes visibly propagate across the simulated watershed. The prototype exhibit's focus on interactivity and exploration, as well as its real-time visual feedback, are designed to foster cause-and-effect reasoning and systems thinking.

\section{Museum Study}

To investigate learning and engagement with a game-based learning environment in an informal setting, a study was conducted with 43 summer campers, ranging in age from $8-14$, at a science museum. Participants were grouped into separate cohorts divided across three sessions $(\mathrm{N}=14,16,13)$. Pre-test measures were administered on the first day of each cohort's week-long summer camp. The pre-test consisted of a demographic survey and three complementary measures of sustainability understanding: a personal meaning map, a sustainability identification task, and a sustainability image-sorting task.

Personal meaning maps (PMMs) consisted of a blank piece of paper with a brief set of instructions and a prompt phrase: sustainability. Participants used a pen to write or draw words, phrases, and pictures about their conceptualizations of the prompt phrase. After the learning experience, participants could revise their PMMs with a different colored pen. After the study, two raters scored each PMM based on the relevance and accuracy of each element on the page. Inter-rater reliability for the pretest $(r=.84)$ and post-test $(r=.88)$ PMMs achieved high levels.

For the identification task, learners inspected an illustrated picture of an environment-depicting both sustainable and unsustainable environmental practices - and annotated the picture by circling "good" practices and crossing out "bad" practices. Participants revised their annotations during the post-test. Two raters scored the annotations using a rubric vetted by subject matter experts, and achieved high agreement on both the pre-test $(r=.97)$ and post-test $(r=.95)$.

For the image-sorting task, learners were given copies of ten images depicting various environmental practices (e.g., solar panels, traffic congestion). Participants organized the images into two categories of their choosing, with the goal or choosing two categories containing as similar a number of images as possible. An expert-based categorization of "sustainable" and "unsustainable" was considered the gold standard response, and this benchmark was used to grade responses.

Later in the study, several days after the pre-test, participants were given the opportunity to explore various parts of the museum, including an area where the FUTURE WORLDS exhibit was located. During these study sessions, all participants 
entered the study room at the same time and were allowed to spend up to 40 minutes in the area. Learners could leave the space at anytime and were free to explore as they saw fit. However, once they left the area, learners were not permitted to return.

Including FUTURE WORLDS, there were 13 exhibits in the study area. No other museum visitors had access to the room during the study. A nearby human docent was available to resolve technical issues and answer questions. Verbal interactions between the docent and participants were otherwise kept to a minimum. Eleven of the exhibits in the study room were permanent exhibits at the museum. In addition to FUTURE WORLDS, one temporary exhibit was added to serve as a control. This temporary exhibit was the only other exhibit with a human docent, and it consisted of a white board with a sign asking learners to share the most interesting thing they learned in the citizen science area using sticky notes. None of the content in the distractor exhibits overlapped with FUTURE WORLDS.

A post-test was conducted immediately following each participant's exit of the study area, which included the same sustainability measures as the pre-test. All sessions were video recorded. Post-study analyses of the video data were conducted by two coders to determine total dwell time (time spent interacting with the exhibit to any extent) as well as time spent in each of three possible "tiers of proximity" relative to FUTURE WORLDS. Inter-rater reliability was established with a subset of the study data and then the remainder was coded independently $(\mathrm{k}=.70)$.

\section{Empirical Findings}

To investigate the efficacy of the FUTURE WORLDS exhibit, statistical analyses of the pre- and post-test measures, as well as coded video recordings, were conducted. Paired t-tests indicated that learners showed significant gains in PMM score from pretest $(M=0.8, S D=1.8)$ to post-test $(M=1.2, S D=2.3), t(37)=2.5, p<.05$. There were also significant increases on the identification task from pre-test $(M=5.96, S D=$ $2.45)$ to post-test $(M=6.42, S D=2.55), t(37)=3.28, p<.05$, as well as significant gains on the image sorting task from pre-test $(M=7.11, S D=3.81)$ to post-test $(M=$ $8.66, S D=2.67), t(37)=2.59, p<.05$. Correlation analyses were conducted to investigate whether learners' individual characteristics - including age and gendershowed significant relationships with learning outcomes, but none were observed.

For each learner, total dwell time, as well as time spent in each of three proximity tiers, was determined using video recording data. Across all participants, the average dwell time at FUTURE WORLDS was 12.5 minutes. This is promising, given dwell times typically reported in other informal contexts, such as 5.03 minutes [5] and 4.9 minutes [7]. However, it should be noted that FUTURE WORLDS dwell times were recorded in a semi-controlled study setting, whereas the above cited dwell times were recorded from observations of the general public in non-controlled settings.

A two-tailed independent samples t-test revealed a significant effect of gender on dwell time, where girls $(M=8 \mathrm{~m}: 46 \mathrm{~s}, S D=5 \mathrm{~m}: 21 \mathrm{~s})$ spent roughly half the time as boys $(M=16 \mathrm{~m}: 23 \mathrm{~s}, S D=10 \mathrm{~m}: 33 \mathrm{~s})$ engaging with FUTURE WORLDS, $t(35)=2.86, p$ $<.05$. To follow up on this analysis, tier-specific dwell time was examined by gender. Results indicated that males $(M=14 \mathrm{~m}: 12 \mathrm{~s}, S D=7 \mathrm{~m}: 34 \mathrm{~s})$ spent significantly more 
time in the first tier than females $(M=8 \mathrm{~m}: 56 \mathrm{~s}, S D=3 \mathrm{~m}: 56 \mathrm{~s})$, which is the tier of closest proximity to the exhibit, $t(29)=2.27, p<.05$.

Additional findings about the influence of learners' individual characteristics emerged from analyses of engagement based on learner age. Results indicated that younger children spent more time at the FUTURE WORLDS exhibit than older children, $t(44)=3.52, p<.01$. In fact, children under age 10 spent twice as much time $(M=$ $20 \mathrm{~m}: 50 \mathrm{~s}, S D=12 \mathrm{~m}: 33 \mathrm{~s})$ as children age 10 and older $(M=9 \mathrm{~m}: 56 \mathrm{~s}, S D=6 \mathrm{~m}: 45 \mathrm{~s})$.

\section{Discussion}

Results suggest that learners' sustainability understanding improves from interactions with FUTURE WORLDS. Furthermore, evidence of extended dwell time suggests that learners are highly engaged with the exhibit. In combination, these two sets of findings suggest that learner engagement with FUTURE WORLDS is not superficial; learners are actively engaged for prolonged periods at sufficient depth to yield significant learning gains across three distinct measures of sustainability knowledge. Also, FUTURE WORLDS appears to be equally effective for boys and girls, as well as young and older children, in terms of fostering learning.

Our observation that gender and age have significant effects on dwell time point toward engagement-centric design implications for future iterations of FUTURE WORLDS and intelligent game-based learning environments in general. Regarding gender, several possible mediating factors could explain why girls spent less time with FUTURE WORLDS than boys, such as video game interest or affinity for the game's visual aesthetic style. Additional studies are necessary to isolate what design factors are responsible for the observed gender differences, and how elements of FUTURE WORLDS' design should be augmented to deliberately appeal to females to an extent that is comparable to males. Regarding age, results suggest that future iterations of FUTURE WORLDS should incorporate problem-solving scenarios that span a broader range of content and complexity. It is plausible that the implemented problem scenarios in the FUTURE WORLDS prototype were sufficiently challenging for young children but were not difficult enough for older children, and thus did not sustain their engagement for extended periods. Incorporating intelligent tutoring system capabilities to dynamically adapt the difficulty of problem scenarios to individual learners, or groups of learners, is a promising way to match scenarios' content complexity to the capabilities of diverse learners. We hypothesize that this form of adaptive pedagogical functionality will increase motivation and dwell time. However, adaptive pedagogical planning will require models for automatically detecting learners' individual characteristics as they approach and use exhibits. Administering lengthy pre-tests is not a viable design choice for most informal settings; automated detector models show more promise for diagnosing learner characteristics to inform adaptive pedagogical functionalities.

Notably, we did not find a relationship between dwell time and learning. We expect that adding curricular material beyond the prototype's current proof-of-concept scope - creating opportunities for more variance in content exposure - could reveal relationships between dwell time and learning that have not thus far been observed. 


\section{Conclusions and Future Work}

Intelligent game-based learning environments show considerable promise for informal settings such as museums and science centers. Creating effective intelligent game-based learning environments for museums requires a grounded understanding of how learners engage with, and learn from, game-based exhibits. In this paper, we described an empirical study that found FUTURE WORLDS yields significant gains in sustainability knowledge, as well as high levels of engagement, during museum learning. Furthermore, boys were observed to actively engage with FUTURE WORLDS for significantly longer durations than girls, and young children engaged with the exhibit for longer periods than did older children. These individual differences underscore the importance of future work investigating adaptive pedagogical functionalities, as can be provided by intelligent tutoring techniques, to dynamically tailor game-based learning experiences based on gender and age, as well as automated detectors for diagnosing learners' individual and group characteristics.

\section{Acknowledgements}

This research was supported by the National Science Foundation under Grant DRL1114655. Any opinions, findings, and conclusions or recommendations expressed in this material are those of the authors and do not necessarily reflect the views of the National Science Foundation.

\section{References}

1. Clark, D., Tanner-Smith, E., Killingsworth, S., Bellamy, S.: Digital Games for Learning: A Systematic Review and Meta-Analysis (Executive Summary). SRI International, Menlo Park, CA (2013)

2. Jackson, G.T., McNamara, D.S.: Motivation and Performance in a Game-based Intelligent Tutoring System. J. Educ. Psychol. 105, 1036-1049 (2013)

3. Shute, V.J., Ventura, M., Kim, Y.J.: Assessment and Learning of Qualitative Physics in Newton's Playground. J. Educ. Res. 106, 423-430 (2013)

4. Johnson, W.L.: Serious Use of a Serious Game for Language Learning. Int. J. Artif. Intell. Educ. 20, 175-195 (2010)

5. Lane, H., Cahill, C., Foutz, S., Auerbach, D., Noren, D., Lussenhop, C., Swartout, W.: The Effects of a Pedagogical Agent for Informal Science Education on Learner Behaviors and Self-efficacy. In: 16th International Conference on Artificial Intelligence in Education, pp. 309-318 (2013)

6. Rowe, J.P., Lobene, E. V., Mott, B.W., Lester, J.C.: Play in the Museum: Designing Game-Based Learning Environments for Informal Education Settings. Proceedings of the 9th International Conference on Foundations of Digital Games (2014)

7. Horn, M.S., Solovey, E.T., Crouser, R.J., Jacob, R.J.K.: Comparing the use of tangible and graphical programming languages for informal science education. In: SIGCHI Conference on Human Factors in Computing Systems, pp. 975-984. ACM Press, New York (2009) 